\title{
STEEPENING OF AFTERGLOW DECAY FOR JETS INTERACTING WITH STRATIFIED MEDIA
}

\author{
PAWAN KUMAR \\ IAS, Princeton, NJ 08540 \\ Alin PANAitescu \\ Dept. of Astrophysical Sciences, Princeton University, NJ 08544
}

\begin{abstract}
We calculate light-curves for Gamma-Ray Burst afterglows when material ejected in the explosion is confined to a jet which propagates in a medium with a power-law density profile. The observed light-curve decay steepens by a factor of $\Gamma^{2}$ when an observer sees the edge of the jet. In a uniform density medium the increase in the power-law index $(\beta)$ of the light-curve as a result of this edge effect is $\sim 0.7$ and is completed over one decade in observer time. For a pre-ejected stellar wind $\left(\rho \propto r^{-2}\right) \beta$ increases by $\sim 0.4$ over two decades in time due to the edge effect and the steepening of the light-curve due to the jet sideways expansion takes about four decades in time. Therefore, a break in the light-curve for a jet in a wind model is unlikely to be detected even for very narrow jets of opening angle of a few degrees or less, in which case the lateral expansion occurs at early times when the afterglow is bright.

The light-curve for the afterglow of GRB 990510, for which an increase in $\beta$ of approximately 1.35 was observed on a time scale of 3 days, cannot be explained only by the sideways expansion and the edge effects in a jet in a uniform ISM - the increase in $\beta$ is too large and too rapid. However, the passage of the cooling or synchrotron peak frequencies through the observing band at about $0.1-1$ day together with jet edge effect explains the observed data. The jet opening angle is found to be $\sim 5^{\circ}$ and the energy in the explosion to be less than about $10^{50} \mathrm{erg}$.
\end{abstract}

Subject headings: gamma rays: bursts - gamma-rays: theory

\section{INTRODUCTION}

In a recent paper Chevalier and Li (1999) pointed out that some of the GRB afterglow light-curves are best modeled when the density of the circum-burst medium is taken to fall off as $r^{-2}$ (this is referred to as the wind model). These afterglows show no evidence for a jet, i.e. their light-curves follow a power-law decline without any break. This is puzzling since collimated outflows are expected in the collapsar model for GRBs (MacFadyen, Woosley \& Heger 2000). We offer a possible explanation for this puzzle by showing that the light-curve resulting from the interaction of a jet with a pre-ejected wind falls off as a power-law whose index changes very slowly with time.

We carry out a detailed modeling of the multi-wavelength afterglow flux data for GRB 990510, which provides the best evidence for a jet propagation in a uniform density medium (Harrison et al. 1999, Stanek et al. 1999), to show that effects associated with a finite jet opening-angle are insufficient to explain the observed rapid steepening of the light-curve.

In $\$ 2$ we calculate the propagation of a jet in a stratified medium and in $\$ 3$ we describe the calculation of the synchrotron emission and afterglow light-curve.

\section{DYNAMICS OF EXPANDING JETS}

The dynamical evolution of jets and its synchrotron emission have been previously investigated by a number of people, e.g. Rhoads (1999), Panaitescu \& Mészáros (1999), Sari, Piran \& Halpern (1999), Moderski, Sikora, \& Bulik (2000), Huang et al. (2000). The evolution of the Lorentz factor $(\Gamma)$ can be calculated from the following set of equations

$$
\frac{d M_{1}}{d r}=2 \pi A r^{2-s}(1-\cos \theta)
$$

$$
\begin{gathered}
\frac{d \theta}{d r}=\frac{1}{f r\left(\Gamma^{2}-1\right)^{1 / 2}}+\frac{\Theta-\theta}{r}, \\
M_{0} \Gamma+M_{1}\left(\Gamma^{2}-1\right)=M_{0} \Gamma_{0},
\end{gathered}
$$

where $\theta$ is the half-opening angle of the jet, $M_{0}$, and $\Gamma_{0}$ are the initial mass and Lorentz factor of the ejecta, $M_{1}$ is the swept-up mass, $\rho(r)=A r^{-s}$ is the density of the circum-stellar medium, and $f=c / c_{s}$ is the ratio of the speed of light to that of the jet sideways expansion; $f$ is a parameter of order unity whose effect can be absorbed in $\Gamma_{0}$, and which has little effect on the light-curve. $\Theta$ is the angle between the velocity vector at the jet edge and the jet axis (in the lab frame) and is determined by the modification of particle trajectory due to the sideways expansion. The last equation above expresses the conservation of energy and it applies to an adiabatic shock when the heating of the original baryonic material of rest mass $M_{0}$ by the reverse shock is ignored.

The above equations can be combined and rewritten in the following non-dimensional form which is applicable for relativistic as well as non-relativistic jet dynamics

$$
\begin{gathered}
\frac{d y_{1}}{d x}=-\frac{x^{2-s}\left(y_{1}^{2}-\Gamma_{0}^{-2}\right)^{2} y_{2}^{2}}{2 y_{1}-y_{1}^{2}-\Gamma_{0}^{-2}}, \\
\frac{d y_{2}}{d x}=\frac{1}{f \theta_{0} \Gamma_{0} x\left(y_{1}^{2}-\Gamma_{0}^{-2}\right)^{1 / 2}}+\frac{\Theta-\theta}{x \theta_{0}}
\end{gathered}
$$

where $x=r / R_{d a}, y_{1}=\Gamma / \Gamma_{0}, y_{2}=\theta / \theta_{0}$, and

$$
R_{d a}=\left(\frac{E}{\pi A c^{2} \theta_{0}^{2} \Gamma_{0}^{2}}\right)^{1 /(3-s)}
$$


is the deacceleration radius. $E=M_{0} \Gamma_{0}$ is the energy in the explosion and $\theta_{0}$ is the initial half-opening angle of the jet. The above equations show that for the wind and the uniform ISM models $\Gamma \propto t_{\text {obs }}^{-1 / 4} \& t_{o b s}^{-3 / 8}$, respectively, as long as $\Gamma \gg \theta_{0}^{-1}$, where $t_{o b s}=\int d t(1-v)$ is the observer time, $t$ being the lab frame time and $v$ the jet velocity in units of $c$.

Equations (4) and (5) are solved, subject to the boundary conditions $y_{1}=y_{2}=1$ for $x \ll 1$, to determine $\Gamma$ and $\theta$ as functions of $r$. For a relativistic jet with $\Theta=\theta$, i.e. fluid velocity in the radial direction, equation (5) reduces to

$$
\frac{d y_{1}}{d x}=-\frac{x^{2-s} y_{1}^{3} y_{2}^{2}}{2-y_{1}}, \quad \frac{d y_{2}}{d x}=\frac{1}{f\left(\theta_{0} \Gamma_{0}\right) x y_{1}} .
$$

The solution of the equations (4) and (5) is a two-parameter family of functions, however in the relativistic case the solution depends only on the product $\theta_{0} \Gamma_{0}$.

One can solve equation (7) approximately, ignoring the very early time behavior, to determine the time when the sideways expansion alters significantly the jet dynamics. The two relations in equation (7) can be combined to yield a first order differential equation for $y_{1} y_{2} \equiv y$ which is given by

$$
\frac{d y}{d \xi} \approx-\frac{y^{3}}{2}+\frac{1}{\eta \xi}
$$

with $\eta=f(3-s)\left(\theta_{0} \Gamma_{0}\right)$, a constant, and $\xi=x^{3-s} /(3-s)$. An approximate solution to this equation is

$$
y \approx \frac{1}{2 \xi^{1 / 2}}+\left(\frac{2}{\eta \xi}\right)^{1 / 3} .
$$

Thus, $y \propto \Gamma \theta$ decreases monotonically with radius or time. The transition to jet sideways expansion starts when the two terms in the above equation become equal, i.e. $\xi \sim(\eta / 16)^{2}$, and lasts for an interval in $\xi$ for which $y$ decreases by a factor of $\sim 3$, or $x$ increases by a factor of $\sim 3^{3 /(3-s)}$. The Lorentz factor continues to fall during the transition by a factor of a few. Therefore, the transition time divided by the time at the start of the transition (in observer frame), during which $\alpha_{1} \equiv-d \ln (\Gamma-1) / d \ln t_{o b s}$ increases from $(3-s) /(8-2 s)$ to approximately $1 / 2$, is approximately $9 \times 3^{3 /(3-s)}$. The solution to $y_{1}$ and $y_{2}$ can be obtained by inserting the expression for $y$ into equation (7). However, $y_{1}$ and $y_{2}$ determined this way have much larger error than $y$ and should not be used for any serious calculation.

We solve equations (4) and (5) numerically and show the results for $x\left(t_{o b s}\right)$ and $\alpha_{1}\left(t_{o b s}\right)$ in Figure 1. Note that the change to $\alpha_{1}$ from one asymptotic value, corresponding to spherical shell expansion, to another, when sideways expansion is well underway, takes a long time; the ratio of the final to the initial time for a change in $\alpha_{1}$ of 0.1 for a uniform ISM is $\sim 10^{2}$ whereas for $s=2$ the ratio is $10^{3}$. For the parameters chosen here $\alpha_{1}=0.5$ when $\Gamma$ is of order a few. In the non-relativistic phase of the jet expansion $\alpha_{1}=1.2$, as for a Sedov-Taylor spherical shock wave.

\section{SYNCHROTRON EMISSION FROM RELATIVISTIC JETS}

The synchrotron spectrum in the co-moving frame is taken to be a sequence of power-laws with breaks at the self-absorption, synchrotron peak, and cooling frequencies, as presented in Sari, Narayan \& Piran (1998); these frequencies can be found in eg. Panaitescu \& Kumar (2000). All of our numerical results, unless otherwise stated, are obtained by integrating emission over equal arrival time surface. Ignoring the radial structure of the jet, the flux received by an observer located on the jet axis is given by

$$
f_{\nu}\left(t_{o b s}\right)=\frac{1}{8 \pi d^{2}} \int_{r_{\min }}^{r_{\max }} \frac{P_{\nu^{\prime}}^{\prime}(r)}{\gamma^{3}\left[1-v \cos \psi\left(r, t_{o b s}\right)\right]^{2}} \frac{d r}{r},
$$

where $P_{\nu^{\prime}}^{\prime}$ is the co-moving power per frequency at $\nu^{\prime}=$ $\gamma(1-v \cos \psi) \nu, r \cos \psi=c t-c t_{o b s}$ and $r_{\text {min }}$ and $r_{\text {max }}$ are solutions of

$$
c t\left(r_{\max }\right)-r_{\max }=c t\left(r_{\text {min }}\right)-r_{\text {min }} \cos \theta\left(r_{\text {min }}\right)=t_{o b s} .
$$

We ignore the angular integration when discussing the analytical calculation of the observed flux and its power-law decline with time. The observed flux at a frequency that is greater than both the cooling frequency, $\nu_{c}$, and the synchrotron peak, $\nu_{m}$, is proportional to

$$
f_{\nu} \propto t_{o b s}^{\frac{1}{2}(4-s)-\frac{1}{4} s p} \Gamma^{\frac{1}{2}(p+2)(4-s)} \min \left\{\left(\theta_{0} \Gamma_{0}\right)^{-2}, y^{2}\right\} .
$$

At early times when $\Gamma \gg \theta^{-1}$ and $\Gamma \propto t_{\text {obs }}^{-(3-s) /(8-2 s)}$, the flux decays as $t_{\text {obs }}^{-(3 p-2) / 4}$. At late times when $\Gamma \theta \lesssim 1$ the powerlaw index for the flux $\beta \equiv-d \ln f_{\nu} / d \ln t_{o b s}=(4-s)\left[\alpha_{1}(p+\right.$ $2)-1] / 2+s p / 4+\alpha_{2}$, where $\alpha_{2} \equiv-2 d \ln y / d \ln t_{o b s}$.

There are two effects that determine the evolution of $\beta$. One of them, the edge effect, is purely geometrical and results from the angular opening $\sim \Gamma^{-1}$ of the relativistic observing cone becoming larger than the jet opening angle $\theta$, i.e. the observer "sees" the edge of the jet. The increase to $\beta$ resulting from it is $\alpha_{2} \lesssim(3-s) /(4-s) ; \alpha_{2}$ decreases with time and therefore the jump in $\beta$ is smaller for larger $\theta_{0}$. The dimensionless time for $\beta$ to increase by $\alpha_{2}$ depends on the angular position of the observer w.r.t. the jet axis and is approximately the ratio of the time when the observer sees the far edge of the jet to the time when the near side of the jet becomes visible. This time is given by

$$
R_{t_{e}} \sim\left[\frac{\theta_{0}+\phi_{0}}{\theta_{0}-\phi_{0}}\right]^{(8-2 s) /(3-s)}=\left[\frac{1+P_{\phi_{0}}^{1 / 2}}{1-P_{\phi_{0}}^{1 / 2}}\right]^{(8-2 s) /(3-s)},
$$

where $P_{\phi_{0}}$ is the probability that the observer lies within an angle $\phi_{0}$ of the jet axis. For $P_{\phi_{0}}=0.25, R_{t_{e}}$ is 18.7 (81) for $s=0$ (2), and during this time $\beta$ increases by approximately $0.7(0.4)$. The dependence of $R_{t_{e}}$ on $\phi_{0}$ becomes much weaker when the emission is integrated over equal arrival time surface (Figure 2). This is because the effect of angular integration is to smear the jet-edge by an angle $1 / \Gamma \sim \theta_{0} / 2$, which sets the minimum value of $R_{t_{e}}$ to be about $10\left(10^{2}\right)$ for uniform (wind) models.

The other effect which leads to a steepening of the afterglow decay is dynamical and is caused by the lateral spreading of the jet. During the relativistic phase the increase to $\beta$ from the sideways expansion is $\delta \beta=(p+2)(4-s) \delta \alpha_{1} / 2+\delta \alpha_{2}$; $\delta \alpha_{1}$ and $\delta \alpha_{2}$ can be read from Figure 1. Since $\alpha_{1}$ does not asymptote to 0.5 so $\beta \neq p$ during the relativistic sideways expansion of the jet. ${ }^{1}$ The value of $\beta$ does, however, approach

${ }^{1}$ It should be noted that the asymptotic behavior $\beta \rightarrow p$ for $s=0$ (Rhoads 1999) is achieved only for extremely narrow jets ( $\theta_{0} \lesssim 1^{o}$ ), so that the jet remains

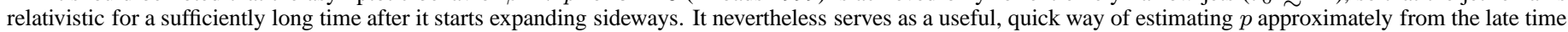
light-curve, when $\beta$ is no longer increasing. 
$p$ because $\delta \alpha_{1} \approx 1 /(8-2 s)$ sometime before the jet becomes non-relativistic and $\alpha_{2} \approx 0$ at this time, thereby giving $\beta \approx p$ (see eq. [12] and Figures $1 \& 2$ ); $\beta$ can exceed $p$, as can be seen in Figure 2, however the decrease in $\alpha_{2}$ during the mildly relativistic phase prevents $\beta$ from getting much larger than $p$. This result can be extended to any observing frequency $\nu>\nu_{m}$ after an appropriate modification of equation $(12$ ). For instance, to consider the case of $\nu_{c}>\nu>\nu_{m}$ the right side of the equation should be multiplied by a factor of $\left(t_{o b s} \Gamma^{2}\right)^{(1-3 s / 4)}$, which has little effect on the evolution of $\beta$. The time scale for the increase in $\beta$ due to sideways expansion is of order $10^{2}\left(10^{3}\right)$ for $\mathrm{s}=0$ (2) (see Figure 2). Therefore this effect is smaller than that resulting from seeing the jet edge, and it extends over a much longer time.

To conclude, we wish to emphasize that for most jets propagating in a uniform ISM we are likely to see an increase to $\beta$ of only $0.6-0.9$; the remainder of the increase takes place on a long time scale, and thus is hard to detect. For jets in a windy medium, $s=2, \beta$ changes by less than about 0.5 and the transition time $R_{t_{e}} \sim 10^{3}$. Such a gradual increase to the afterglow light-curve power-law index is extremely difficult to detect (see Figure 2). For instance, if the edge of the jet becomes visible at $t_{\text {obs }} \sim 1$ day, the difference in the optical flux at the end of 10 days with and without jet is $\sim 0.25 \mathrm{mag}$, which can be easily missed. Thus, the GRBs studied by Chevalier and Li (1999), which show evidence for the wind model, could in fact have had a collimated ejection of material.

\subsection{The Afterglow of GRB 990510}

The optical emission of the afterglow of GRB 990510 was measured in the V, R and I bands between 0.15 and 7 days after the burst and showed the power-law index of the light-curve, $\beta$, to have increased from $0.82 \pm 0.02$ to $2.18 \pm 0.05$ (Harrison et al. 1999) or from $0.76 \pm 0.01$ to $2.40 \pm 0.02$ (Stanek et al. 1999) during a dimensionless time $R_{t_{e}} \approx 30$ which, as described previously, is not possible to obtain through the effects of the jet sideways expansion alone. Therefore there must be some contribution to the light-curve steepening due to the passage of one (or both) of the spectral breaks: the synchrotron peak $\nu_{m}$ and the cooling frequency $\nu_{c}$.

In Figure 3 we show a comparison between the light-curves of GRB 990510 in the $V, R, I$ bands and the $8.7 \mathrm{GHz}$ radio data, with a model where the cooling frequency $\nu_{c}$ crosses the optical band at $t_{o b s} \sim 1$ day. The steepening of the light-curve has little dependence on the observing band because the ratio of the largest to the smallest optical wavelength is $\sim 1.5$. Moreover, the integration over angle spreads in time the steepening of $\beta$, making it nearly achromatic. An increase of $\beta$ by $\sim 0.8$ is caused by the jet edge and the sideways expansion, and an increase of 0.25 results from the passage of $\nu_{c}$ through the observing band. A further increase of $\beta$ of $\sim 0.15$ is caused by the passage of $\nu_{m}$ through the observing band at $t_{o b s} \sim 0.03$ day (see lower panel of Figure 3); the transition time for $\beta$ to increase by $\sim(3 p-1) / 4$ due to the $\nu_{m}$ crossing is about a decade in the observer frame as a result of integration over equal arrival time surface, hence one should be careful in deducing $p$ from $\beta$ at early times. All these together give rise to a light-curve that is consistent with the data. The model is also consistent with the
HST $V$-band observation carried out at about a month after the burst (Fruchter et al 1999). The parameters for the fit are given in the caption for fig. 3 which yields the energy in the burst to be $2 \times 10^{49} \mathrm{erg}$. Correcting for the radiative losses the energy in the burst increases by a factor of a few to $\lesssim 10^{50} \mathrm{erg}$. We estimate the uncertainty in model parameters by varying them in such a way that the numerically calculated light-curve lies within 3- $\sigma$ of the observed data points. We find the uncertainty in the jet opening angle and the burst energy to be a factor of two, and $\epsilon_{e}, n$ and $\epsilon_{B}$ are found to be uncertain by factors of about 4, 40 and 7 respectively; we note that the radio observations are very important in constraining the model parameters. The electron index $p$ is constrained by the observed $\beta$ before and after the $\sim 1$ day break; the error in $p$ is $\sim 5 \%$.

The optical emission of the afterglow of GRB 990510 can also be explained by a model where the synchrotron peak frequency crosses the observed band at $\sim 0.1$ day. Its effect on $\beta$ persists for up to $\sim 1$ day and yields an increase of $\beta$ of $\sim 0.5$ during the early observations. The parameters for the second model differ from the one described above (see Figure 3 ) somewhat. In particular, $\epsilon_{e}$ is larger by a factor of two, the energy per solid angle is smaller by a factor of two, and $\theta_{0}$ is $\sim 20 \%$ larger.

\section{CONCLUSIONS}

One of the main results of this work is to show that afterglows from well collimated Gamma-Ray Burst remnants going off in a medium with density decreasing as $r^{-2}$ show little evidence for light-curve steepening due to jet edge and sideways expansion. This could explain the lack of breaks in the afterglows of GRB 980326 and GRB 980519, which Chevalier \& Li (1999) found to offer support for the wind model. Jets can perhaps be detected by the measurement of time dependent polarization.

In a collimated outflow the sharpest break in the light-curve is produced in a uniform density circum-stellar medium, and is associated with the edge of the jet coming within the relativistic beaming cone (the edge effect). The magnitude of this break is $\sim 0.7(0.4)$ for a uniform ISM (wind model) and occurs over about 1 decade ( 2 decades) in time. Further steepening of the light-curve, associated with the sideways expansion of the jet, occurs on a much longer time scale of $R_{t_{e}} \sim 10^{2}\left(10^{4}\right)$, i.e. weeks to months.

The power-law index for the light-curve of GRB 990510 increased between days 0.8 and 3 by about 1.35 . This is too large and too fast to result from jet edge \& sideways expansion effects. However, the observations can be explained if either the cooling or the synchrotron peak frequency passed through the observing band at about 1 or 0.1 day, respectively. Models that are consistent both the optical and radio data of this afterglow have an opening angle of $\sim 5^{\circ}$ and energy in the explosion is $\lesssim 10^{50}$ erg (see Figure 3 ).

For the afterglow of GRB 990123 the power-law index of the light-curve increased by 0.55 between days 1.5 and 3 , which can be explained by the edge effect alone (Mészáros \& Rees 1999).

We thank Peter Mészáros and Vahe Petrosian for useful discussions. 


\section{REFERENCES}

Beuermann, K., Reinsch, K., \& Hessman, F. 1999, GCN \#331

Chevalier, R.A., and Li, Z.-Y. 1999, 520, L29

Fruchter, A. et al. 1999, GCN \#386

Harrison, F., et al. 1999, ApJ, 523, L121

Huang, Y., Dai, Z., \& Lu, T. 2000, A\&A, submitted astro-ph/0002433

Kulkarni, S. et al. 1999, Nature, 398, 389

Marconi, G. et al. 1999, GCN \#329, \#332

MacFadyen, A Woocley, SE, \& Heger, $\triangle 200$ ApI, submitted astro$\mathrm{ph} / 9910034)$

Mészáros , P. \& Rees, M.J. 1999, MNRAS, 306, L39
Moderski, R., Sikora, M., \& Bulik, T. 2000, ApJ, 529, 151

Panaitescu, A. \& Mészáros, P. 1999, ApJ, 526,707

Panaitescu, A. \& Kumar, P. 2000, astro-ph/0003246

Pietrzynski, G. \& Udalski, A. 1999, GCN \#319, \#328

Rhoads, J. 1999, ApJ, 525, 737

Sari, R., Piran, T., \& Halpern, J. 1999, ApJ, 519, L17

Sari, R., Piran, T., \& Narayan, R. 1998, ApJ, 497, L17

Stanek, K., Garnavich, P., Kaluzny, J., Pych, W., \& Thompson, I. 1999, ApJ, 522, L39

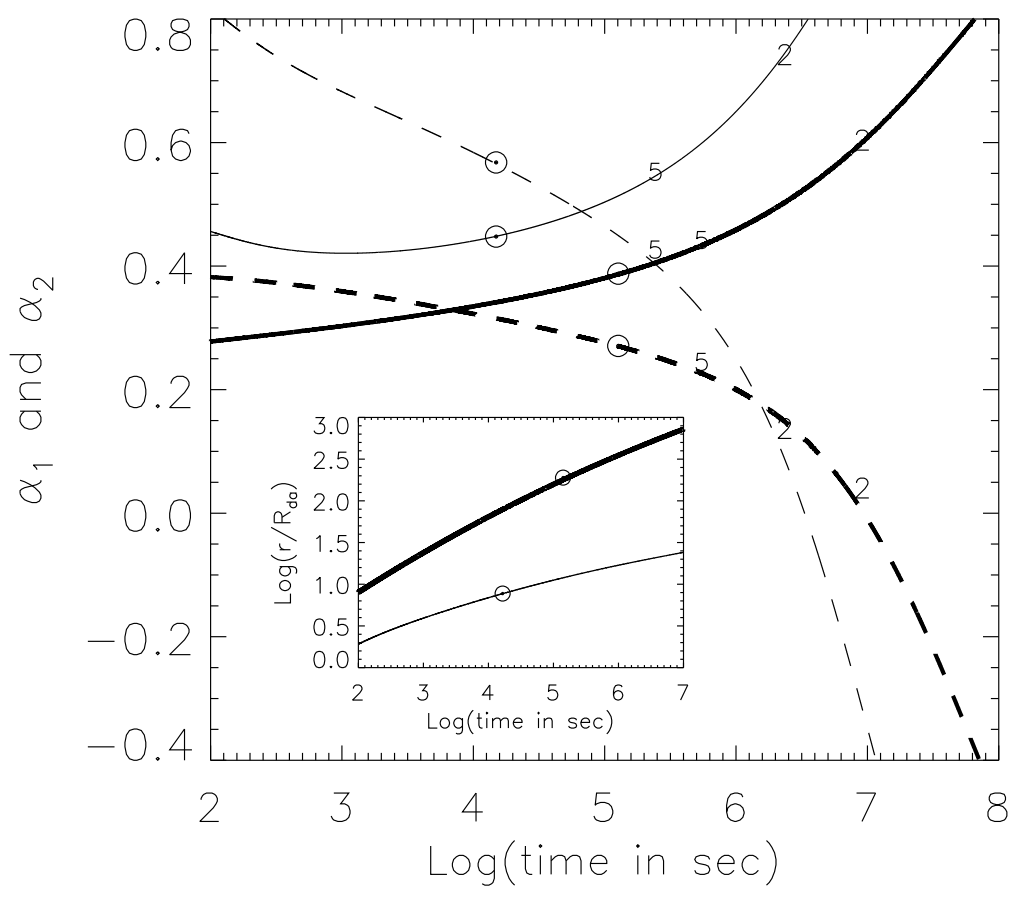

FIG. 1.- The main panel shows $\alpha_{1}=-d \ln (\Gamma-1) / d \ln t_{o b s}$ for uniform ISM $s=0$ (thin continuous curve) and the wind model $s=2$ (thick continuous curve), and $\alpha_{2}=-2 d \ln (\theta \Gamma) / d \ln t_{o b s}$ for $\mathrm{s}=0$ (thin dashed curve) and $\mathrm{s}=2$ (thick dashed curve). The inset shows the evolution of the jet radius. The symbol $\odot$ marks the time when $\theta \Gamma=1$, and the symbols 5 (2) denote the time when $\gamma=5(2)$. For this calculation we took the energy per unit solid angle to be $3 \times 10^{53}$ erg $\mathrm{sr}^{-1}$ and $\theta_{0}=1 / 30 \mathrm{rad}$. The density of the uniform ISM is $1 \mathrm{~cm}^{-3}, A=5 \times 10^{11} \mathrm{~g} \mathrm{~cm}^{-1}$ for $s=2$, and $\Gamma_{0}=300$ (i.e. $\Gamma_{0} \theta_{0}=10$ ). 


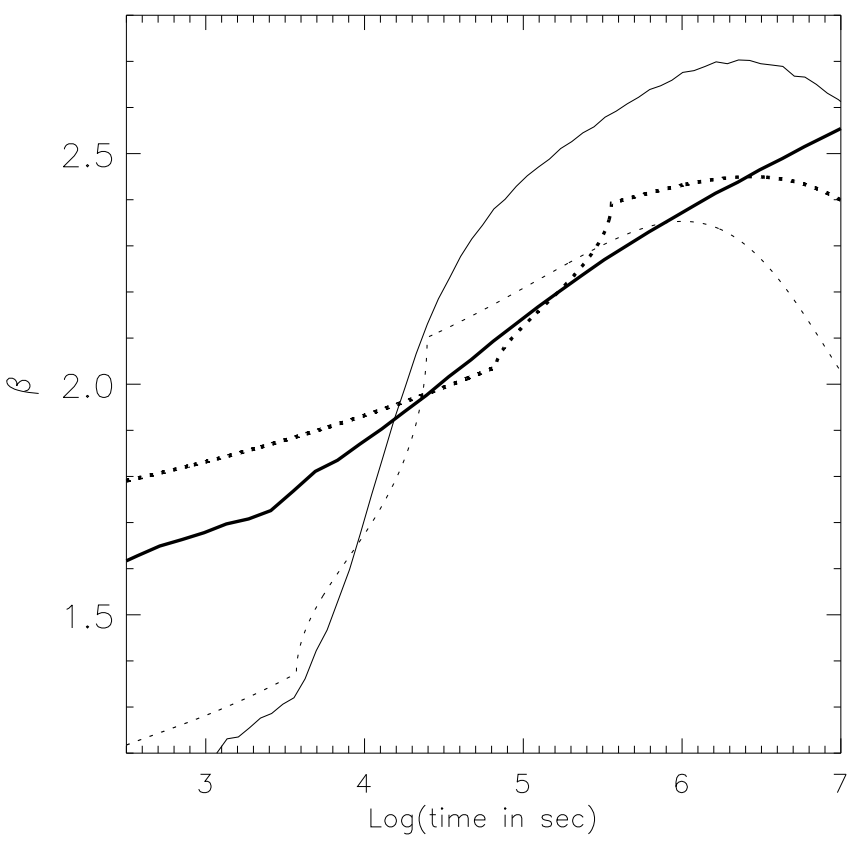

FIG. 2. - The panel shows $\beta \equiv-d \ln f_{\nu} / \ln t_{\text {obs }}$ for $\nu>\max \left(\nu_{m}, \nu_{c}\right)$ and $\phi_{0}=\theta_{0} / \sqrt{5}$. The thin $\&$ thick dashed lines are for $s=0 \& 2$ respectively; the observed flux in these cases was calculated without proper angular integration over the jet surface. The sharp increase to the value of $\beta$ seen in the dashed curves arises from the edge effect described in the text. The thin and the thick continuous curves are for $s=0 \& 2$ respectively and these calculations included integration over equal arrival time surface; note that this smoothes out sharp changes in $\beta$. For all of these calculations we took the energy per unit solid angle to be $3 \times 10^{53}$ erg sr ${ }^{-1}, \theta_{0}=1 / 30 \mathrm{rad}\left(\theta_{0} \Gamma_{0}=10\right), p=2.5$, the density of the uniform ISM is $1.0 \mathrm{~cm}^{-3}$ and $A=5 \times 10^{11} \mathrm{~g} \mathrm{~cm}{ }^{-1}$ for $s=2$. The results shown here are independent on the sideways expansion speed (i.e. the parameter $f$ in eq. [e]), as long as this speed is relativistic. 

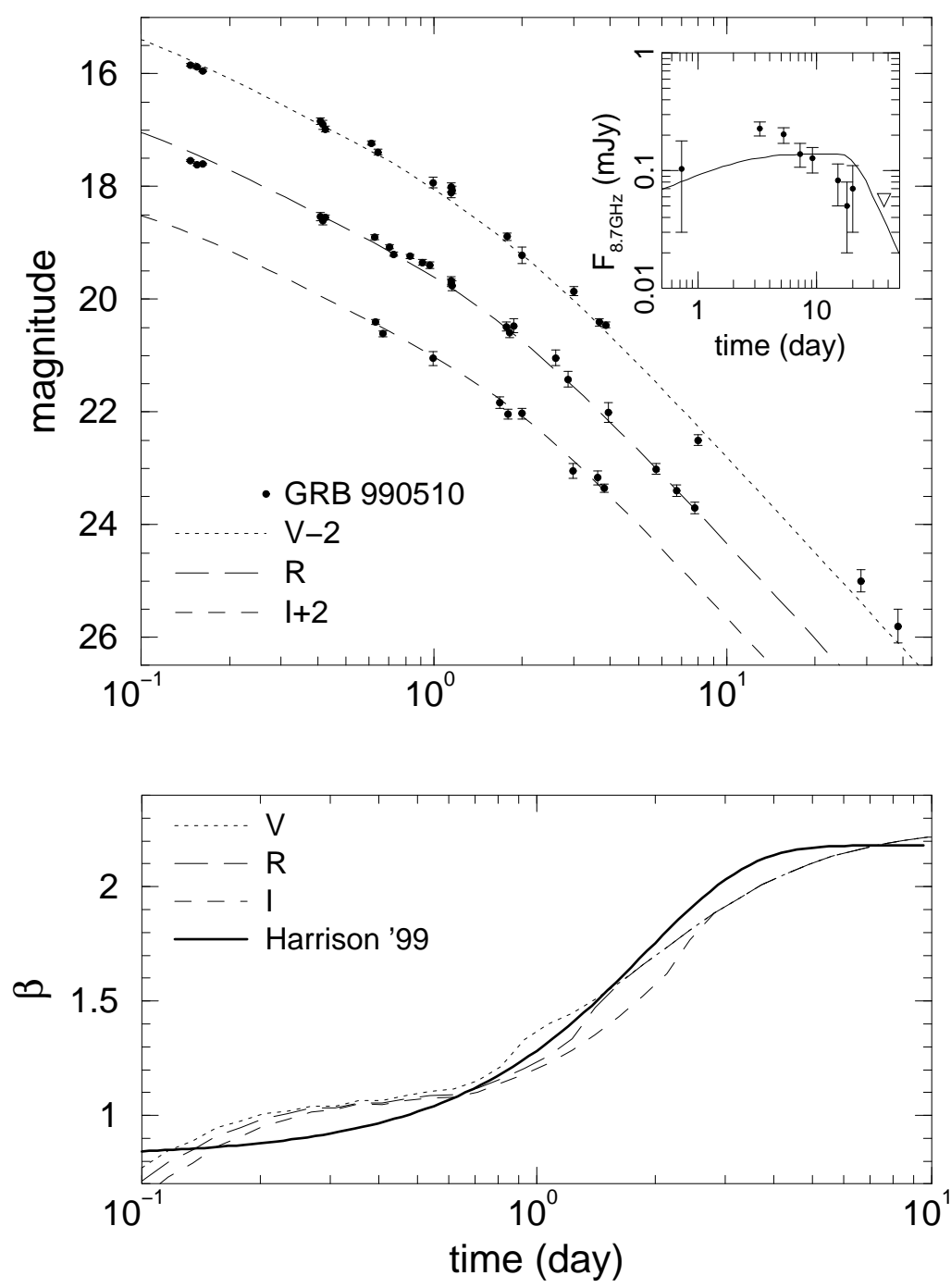

FIG. 3.- Comparison between the observed and the theoretically calculated light-curves for the afterglow of GRB 990510 in the radio and the optical bands _Most of the data is taken from Harrison et al. (1999); the optical data was supplemented with observations reported in GCN Circulars (http://gcn.gsfc.nasa.gov/gcn/gcn3) by Beuermann et al. 1999, Marconi et al. 1999, Pietrzynski \& Udalski 1999; the latest measurements in the $V$-band were taken with the HST (Fruchter et al. 1999). The model light-curves are calculated for an observer located on the jet axis, which gives the fastest decline of the light-curve. The jet has energy per solid angle $E /\left(\pi \theta_{0}^{2}\right)=1.2 \times 10^{52} / 4 \pi \mathrm{erg} \mathrm{sr}^{-1}$ and a half-angle $\theta_{0}=0.085$ rad. The electrons acquire $\epsilon_{e}=0.3$ of the internal energy after shock acceleration, the magnetic field energy is $\epsilon_{B}=0.04$ of that of the shocked gas, and the electron index is $p=2.2$. The external medium is homogeneous with $n=0.23 \mathrm{~cm}^{-3}$. The redshift of the source is $z=1.62$. The cooling frequency passes through the observing window at $t_{o b s}=1.2$ day steepening the afterglow light-curve while the sideways expansion is effective. The inset of the upper panel shows the $8.7 \mathrm{GHz}$ emission. The lower panel shows a comparison of the numerically computed power-law index $(\beta)$ for the decline of the afterglow of GRB 990510 and the observed one, as obtained by the fitting formula given in Harrison et al. (1999). 